pregnancy on general disease and of general disease on pregnancy." These recommendations are capable of different interpretation by individual medical schools, and the Maternity Services Subcommittee of the General Medical Services Committee has expressed concern that some students may receive inadequate training in practical obstetrics. ${ }^{1}$ It would be of interest to know what the students think. Do they corsider themselves competent, on leaving their medical schools, to supervise antenatal care, manage labour, and cope with the commoner complications that may arise before, during, and after delivery of the child? Some are sure to have doubts, yet they can pass through the preregistration year, complete a vocational training programme recognised by the Joint Committee for Postgraduate Training in General Practice, and in time become a principal in general practice without any further training in obstetrics. The point that most concerns the Maternity Services Subcommittee is that all principals in the National Health Service-whether or not they fulfil the criteria for their names to be on the obstetric list-are obliged by the Secretary of State under paragraph 13 of their terms of service to provide care in an obstetric emergency.

Inevitably there must be some comparison with the basic training given to midwives under the direction of the Central Midwives Board. That board has recently decided ${ }^{2}$ that the training period for pupils who are already State-registered Nurses should be extended from 12 to 18 months and that the clinical component should include the antenatal examination of at least 100 women, the delivery of no fewer than 40 women, attendance at no fewer than 40 complicated labours, the examination of 100 mothers during the postnatal period, and the examination of 100 newborn babies, and that pupils should have experience in caring for ill babies and those of low birth weight. It is unlikely that were it to review its recommendations for the teaching of obstetrics in the undergraduate curriculum the General Medical Council would include such precise and extensive directions.

If doctors, especially general practitioners, are to be trained to a standard that will allow them with confidence to step in and help a midwife faced by some emergency then additional and suitable training must be offered in the postgraduate period. It is unrealistic to expect that the preregistration year should always include worthwhile obstetric experience; this would very likely be impossible to arrange and not all doctors would want the experience anyhow. Much better would be agreement about some module of obstetric training, probably coupled with family planning and diseases of women, to be included in all training programmes submitted for recognition to the Joint Committee for Postgraduate Training in General Practice. This at least would ensure that all future principals in general practice had an accepted, basic level of training in obstetrics and closely related subjects. Such an innovation might well affect posts already recognised for training by the Royal College of Obstetricians and Gynaecologists and the implications would need to be discussed with the college and other interested bodies. Whether this component of the vocational training programme should vary depending on whether the doctor wishes to provide a full maternity service or simply undertake antenatal and postnatal care is a point of detail that could well be discussed after the basic principles have been agreed. It is timely that this whole subject should be raised and discussed fully for the present state of affairs is unsatisfactory, embarrassing in some ways, and potentially dangerous.

${ }^{1}$ Anonymous. Strong views on obstetrics. $\mathrm{Br}$ Med $\mathcal{F} 1981$;282:1169-72.

2 Central Midwives Board. Approved training syllabus, September 1980.

\section{Paroxysmal nocturnal haemoglobinuria}

Paroxysmal nocturnal haemoglobinuria is a rare, acquired haemolytic anaemia in which the circulating red blood cells are abnormally sensitive to lysis by activated serum complement. As the name implies, excessive destruction of red cells takes place mainly during the night so that haemoglobinuria is seen only or principally in the early morning. The platelets and granulocytes in paroxysmal nocturnal haemoglobinuria are also abnormally sensitive to complement, and thrombocytopenia or granulocytopenia or both are often seen during the disease.

Paroxysmal nocturnal haemoglobinuria may be diagnosed के simply and reliably by the acidified serum lysis (Ham's) test; $\overrightarrow{0}$ haemosiderinuria is a valuable sign of chronic intravascular haemolysis since iron-containing granules will be present in the urinary sediment even if there is no haemoglobinuria at the time. Haemolysis in paroxysmal nocturnal haemoglobinuria is due to a defect in the erythrocyte membrane. Nevertheless, not all circulating erythrocytes are equally sensitive to activated complement since a considerable population of abnormal cells usually coexist with moderately or normally sensitive red cells. The proportions of normal and abnormal cells in a given patient can best be measured by analysing the curves obtained from complement lysis sensitivity tests. ${ }^{1}$ Thus the severity of haemolysis will depend on both the relative proportions of normal and abnormal cells and the degree of the abnormality. Platelets and granulocytes also interact abnormally with complement, ${ }^{2}$ and, as with the red cells, only some of the granulocytes are defective. ${ }^{34}$ These reactions may partly be responsible for the venous thromboses which occur in patients with paroxysmal nocturnal haemoglobinuria as well as for the chemotactic defect in the granulocytes incubated with activated complement. ${ }^{5}$ Recently Tumen $e t a l^{6}$ have shown that erythroid and myeloid precursor cells also have an increased sensitivity to complement.

Paroxysmal nocturnal haemoglobinuria is a chronic disease, and, if threats such as haemolytic crises and thromboses can be avoided, patients may live for many years. ${ }^{7}$ Venous thrombosis is a frequent problem in patients with paroxysmal nocturnal haemoglobinuria and can cause headache and abdominal pain. ${ }^{89}$ Progressive diffuse hepatic venous thrombosis is one of the most serious complications and a common cause of death. Indeed, abdominal pain in patients with paroxysmal nocturnal haemoglobinuria should be considered secondary to intra-abdominal thrombosis, particularly hepatic venous thrombosis, unless proved otherwise. ${ }^{9}$ Recently Clarke et al $^{10}$ have presented evidence of widespread renal lesions in paroxysmal nocturnal haemoglobinuria, which they attributed to repeated microvascular thrombosis, and no fewer than seven of the 104 patients reviewed by Polli et $\mathrm{l}^{11}$ died from renal failure. Vascular accidents in the brain caused the deaths of three of the eight patients reviewed by Clarke et al ${ }^{10}$ and 12 of the 104 reviewed by Polli et al. ${ }^{11}$ Neutropenia in paroxysmal nocturnal haemoglobinuria results in increased susceptibility to infection, which may be exacerbated by other factors such as saturation of the reticuloendothelial system as a result of haemolysis ${ }^{12}$ or abnormal granulocyte function. ${ }^{2}$

There is good evidence that the abnormal cells in paroxysmal nocturnal haemoglobinuria arise from a defective haemopoietic stem cell, and that normal and abnormal cells coexist in the patient's bone marrow. The clonal origin of the abnormal red cells in a patient with paroxysmal nocturnal haemo. . 
globinuria who was also heterozygous for the two variants of glucose-6-phosphate dehydrogenase (G6PD) was confirmed by finding that the red cells showing the paroxysmal nocturnal haemoglobinuria defect had a single-enzyme phenotype while the normal red cells had a double-enzyme phenotype. ${ }^{13}$ Another patient presented with paroxysmal nocturnal haemoglobinuria and erythroleukaemia and only the neoplastic clone had the paroxysmal nocturnal haemoglobinuria abnormality. ${ }^{14}$ The abnormality in granulocytes is also thought to be clonal. ${ }^{3}$ The paroxysmal nocturnal haemoglobinuria clone may arise in a normal marrow or in one which is already abnormal. The abnormal clone may not persist but may gradually disappear with time and, rarely, patients with paroxysmal nocturnal haemoglobinuria develop aplastic anaemia. Indeed, the similarities between paroxysmal nocturnal haemoglobinuria and aplastic anaemia have been emphasised by Dacie and Lewis ${ }^{15}$ and the myelodysplastic syndromes, including paroxysmal nocturnal haemoglobinuria and aplastic anaemia, share the tendency to transform to acute leukaemia.

Paroxysmal nocturnal haemoglobinuria is usually treated symptomatically. Nevertheless, the only definitive treatments for stem-cell disorders such as paroxysmal nocturnal haemoglobinuria are to alter or replace the abnormal stem-cell population. Stem-cell proliferation has not yet been altered beneficially but normal stem cells can be provided by bone marrow transplantation. A patient with paroxysmal nocturnal haemoglobinuria complicated by refractory marrow failure has been treated successfully by infusion of normal bone marrow. ${ }^{16}$ Since the donor was an identical twin, the patient did not require immunosuppression before receiving the cells but, despite the lack of treatment directed against the abnormal clone, the abnormal cells were displaced by the normal bone marrow. Transplantation of allogeneic bone marrow has also been successful in the treatment of paroxysmal nocturnal haemoglobinuria. ${ }^{1718}$ Nevertheless, at present the hazards of allogeneic bone marrow grafting stand in the way of justifying transplantation as the treatment of choice for patients with paroxysmal nocturnal haemoglobinuria, who may live for years with conventional supportive treatment.

${ }^{1}$ Rosse WF, Dacie JV. Immune lysis of normal human and paroxysmal nocturnal hemoglobinuria $(\mathrm{PNH})$ red blood cells. I. The sensitivity of PNH red cells to lysis by complement and specific antibody. $\mathcal{F}$ Clin Invest $1966 ; 45: 736-48$.

${ }^{2}$ Aster RH, Enright SE. A platelet and granulocyte membrane defect in paroxysmal nocturnal hemoglobinuria: usefulness for the detection of platelet antibodies. $\mathcal{F}$ Clin Invest 1969;45:1199-210.

${ }^{3}$ Brubaker LH, Essig Le RJ, Mengel CE. Neutrophil life span in paroxysmal nocturnal hemoglobinuria. Blood 1977;50:657-62.

4 Stern M, Rosse WF. Two populations of granulocytes in paroxysmal nocturnal hemoglobinuria. Blood $1979 ; 53: 928-34$

${ }^{5}$ Craddock PR, Fehr J, Jacob HS. Complement-mediated granulocyte dysfunction in paroxysmal nocturnal hemoglobinuria. Blood 1976;47: 931-9.

- Tumen J, Kline LB, Fay JW, et al. Complement-sensitivity of paroxysmal nocturnal hemoglobinuria bone marrow cells. Blood 1980;55:1040-6.

${ }^{7}$ Charache S. Prolonged survival in paroxysmal nocturnal hemoglobinuria. Blood $1969 ; 33: 877-83$.

${ }^{8}$ Dacie JV. The haemolytic anaemias: congenital and acquired. Part IV. London: J and A Churchill, 1967.

- Peytremann R, Rhodes RS, Hartmann RC. Thrombosis in paroxysmal nocturnal hemoglobinuria (PNH) with particular reference to progressive, diffuse hepatic venous thrombosis. Series Haematologica $1972 ; 5: 115-36$.

10 Clarke DA, Butler SA, Braren V, Hartmann RC, Jenkins DEJ. The kidneys in paroxysmal nocturnal hemoglobinuria. Blood 1981;57:83-9.

${ }^{11}$ Polli E, Sirchia G, Ferrone S, Mercuriale F, Zanella A. Emoglobinuria parossotica notturna-revisione critica. Milan: Edizione Cilag-Chemie Italiana, 1973.

12 Kaye D, Hook EW. The influence of hemolysis or blood loss on susceptibility to infection. $\mathcal{F}$ Immunol 1963 ;91:65-75.

13 Oni SB, Osunkoya BO, Luzzatto L. Paroxysmal nocturnal hemoglobinuria: evidence for monoclonal origin of abnormal blood cells. Blood 1970 $36: 145-52$

${ }^{14}$ Luzzatto L, Familusi JB, Williams CK, Junaid TA, Rotoli B, Alfinito F. The PNH abnormality in myeloproliferative disorders: association of PNH and acute erythremic myelosis in two children. Haematologica (Pavia) $1979 ; 64: 13-30$

${ }^{15}$ Dacie JV, Lewis SM. Paroxysmal nocturnal haemoglobinuria: variation in clinical severity and association with bone-marrow hypoplasia. Br f Haematol $1961 ; 7: 442-57$.

16 Fefer A, Freeman H, Storb R, et al. Paroxysmal nocturnal hemoglobinuria and marrow failure treated by infusion of marrow from an identical twin. Ann Intern Med 1976;84:692-5.

17 Storb R, Thomas ED, Weiden PL, et al. Aplastic anemia treated by allogeneic bone marrow transplantation: a report on 49 new cases from Seattle. Blood 1976;48:817-41.

${ }^{18}$ Hows JM, Harris R, Palmer S, Gordon-Smith EC. Immunosuppression with cyclosporin $\mathrm{A}$ in allogeneic bone marrow transplantation for severe aplastic anaemia-preliminary results. $\mathrm{Br} \mathcal{F}$ Haematol (in press).

\section{Research in the NHS}

The difficulties encountered by NHS doctors in doing clinical research were the subject of a half-day seminar in Oxford last month, organised by the research committee of the Oxfordshire $\operatorname{AHA}(\mathrm{T})$. The origins of the seminar lay in the growing awareness among doctors in the Oxfordshire area of the difficulty of carrying out good research, and indeed much of the meeting was taken up with a catalogue of problems. Predictably, many of these concerned the inadequacy of resources, particularly time, laboratory space, and the skills of ancillary workers. Speakers also drew attention to NHS doctors' uncertainties about their participation in research, the discouraging attitudes of some senior managers and authority members, and the difficulties that doctors might face in seeking help or collaboration from their local university. While no one suggested that health authorities should assume total responsibility for providing doctors with the resources needed for their research, several speakers argued that a more helpful and encouraging attitude by the authorities would not come amiss.

At a deeper level of analysis the seminar considered the hallmarks of good research and the preconditions for its attainment. Some speakers, particularly those with outstanding records of research achievement themselves, argued that if high standards were to be maintained research workers must be trained properly through early exposure to the rigorous demands of the scientific method and must be insulated from all the diversionary influences that detracted from a serious and dedicated commitment to a research career. Several factors were identified that militated against the training of good researchers in the NHS, including the rigid structures of specialist training that inhibited the development of an inquisitive mind, the sheer pressures of clinical work that often relegated research to the status of a spare-time activity, and the attractions of early promotion to a consultant post.

Other speakers rejected this approach as excessively purist, suggesting instead that research was an integral part of selfeducation and should thus be pursued by all doctors. There was a place, they claimed, for the careful collection and analysis of simple data as well as the theoretical and methodological complexities of advanced research, and the former needed encouraging and funding as much as the latter. The locally organised research scheme operated by the health authorities was regarded as a valuable way of promoting research, not only in providing funds (some $£ 4 \mathrm{~m}$ in the current financial year) but also in exposing research ideas and proposals to the critical appraisal of colleagues. Interestingly, however, the needs and experiences of general practitioners were not raised at all in the 